\title{
Ethical Issues in Enhancement: An Introduction
}

\author{
GLENN McGEE
}

The role of the healer is expanding. Attempts by physicians to enhance human capacity are but one among many new medical projects. The twentieth century ushered in significant changes in therapeutic modalities, and the past two decades have seen the role of the physician reshaped by economic, political, and dramatic new social mores. People ask new and different things of their clinicians. Under managed care, the primary care clinician is expected to have much more skill than was traditionally expected of a general internist, and new incentives force physicians to much more explicitly ration the care they provide to patients and to patient populations. But perhaps no change in the contemporary world of health portends more long-term effects than the introduction of enhancement technologies.

Certain kinds of technologies seem either to expand the healer's role so much or to suggest such dramatic changes in human capacity as to merit the appellation "enhancement medicine." Viagra, the choice of a sperm donor, adolescents' use of Prozac and Ritalin, and an incredible array of cosmetic surgeries have been discussed by commentators, politicians, ethicists, and sociologists in the context of a changing frame of reference for the medical role. ${ }^{1}$ The ability to so radically reshape the human body and mind lies outside the historically rooted understanding of medicine's obliga- tion to cure. That there is dissonance between these new opportunities and the traditional institutions of medicine is suggested by the fact that so few seeming enhancements are covered by insurance or embraced by mainstream clinical practice.

The issues associated with enhancement of human capacity are also raised by more ordinary cases in medicinethreshold cases about how medicine ought to understand, and how far society should pursue the treatment of conditions that are either exceptionally expensive to ameliorate or outside the ordinary range of therapeutic activity. A man who tunes pianos injures his hand in an accident. Physical therapists tell him after months of treatment that his hand has regained as much function as is practical under the circumstances. He tries to return to work, only to find that his hands are no longer capable of tuning pianos. Frustrated, he asks whether it might be possible to return his hands to their prior level of functioning. The therapists respond that although, at a cost of millions of dollars, his hands might be returned to their former prowess, the treatment they have provided to date has resulted in the restoration of normal function, what they would usually consider "a cure." Other routine cases raise questions about how to define medical activity that is not a response to any symptom or illness. Every day in pediatricians' offices 
young children are given inoculation. They are not ill. They are by all applicable clinical standards "healthy." But they are seen and treatment is administered so that they might avoid future diseases. Still other cases raise questions about what kind of therapeutic compromises physicians can or should make in the interest of one or another kind of human flourishing: in clinics and hospitals across the nation, pregnancies are terminated because women believe that the quality of their life will be better if pregnancy ends.

The essays in this special section sketch out different attempts to reconcile enhancement with the ordinary activities of a physician. Several issues are encountered in both the plain enhancements and the borderline cases and several families of concepts have been developed to understand these issues. To introduce the various problems of drawing a distinction between treatment and enhancement, it is essential to identify these families of concepts. They can be split into questions of allocation of medical effort and questions concerning the social and political nature of improving human persons or humanity. In this essay, I introduce four schemes that relate enhancement to medicine.

The first scheme for understanding enhancement is one that originates in the discussion of futility and in attempts to divine an objective standard by which futile treatments could be identified. In this scheme, any appropriate treatment of a patient belongs in one of five simple categories. Therapeutic treatments are those that could ameliorate the disease of a patient or reduce the patient's symptoms in such a way as to halt the physiological progress of the disease. Palliative treatments are those that reduce the symptoms of disease, especially pain, without altering the underlying disease state. When therapy is ceased, such as when a respirator is removed from a patient dying from
ARDS, it is commonly pointed out that "care" has not been stopped, because palliation continues. Evaluative treatments are those that stabilize a patient for diagnosis, or that diagnose. Some treatments do not stabilize for evaluation, palliate, or cure. These, in scheme one, are termed futile, where futile merely denotes the state of affairs in which a treatment is not accomplishing one of those three goals. However, a fourth kind of treatment, the "acceptably futile" treatment, involves a treatment that is "worth" pursuing because an extenuating circumstance presents an agreed-on short-term goal, such as keeping a patient alive long enough to allow a final visit by a relative. The fifth kind of treatment in this scheme is prevention. Preventive care, such as the inoculation of a child, does not fit in any of the other categories for appropriate treatment, and does not, once considered, fit the role of physician as healer of the sick. Preventive care, however, is also not futile in the ordinary sense of futility described above. Those who have argued that there can be discrete categories for clinical activity, and for therapy in particular, hold that prevention is one of the five appropriate activities but is not therapeutic. Enhancement, however, would involve going beyond the prevention of disease and thus beyond the realm of appropriate treatment.

The second scheme for understanding enhancement is rooted in a concept of medical necessity that is widely endorsed but best articulated by Norman Daniels and James Sabin. ${ }^{2}$ Medical necessity entails an ethical obligation of providers and/or payers to provide a service to others (i.e., in private or public insurance systems). Where there is medical necessity, a right to treatment exists. Without medical necessity, the obligation to treat is released and the entitlement to treatment reduced. It is argued that physicians must effectively treat physical or mental disability or 
ameliorate conditions arising from them. Medical services may also effectively produce benefit for other conditions, but in those cases services do not count as medically necessary. Short children not suffering from a disease need not receive growth hormone. Women who lose a breast may have breast reconstruction, but breast augmentation would not be necessary.

In this second scheme, the key to medical necessity is not the amount of suffering caused by a condition, but rather its medical etiology. Treating a shy bipolar person, Daniels reports, is different from treating an unhappy husband because of the use of diagnostics to identify the former as a patient and as a participant in the medical system. The goal of putting so much moral weight on the definition of disease is defended by Daniels and others as a fair way to establish policy based on the moral justification of equality of opportunity. This is important for two reasons: first, it is important to avoid "moral hazard," the modification of behavior in light of incentives from institutions like the insurance industry. Someone with extensive fire insurance may want to make payoff more likely by setting a fire. For this reason, arson is not covered. Speeding tickets are not covered by automobile insurance. If we allow suffering to be included in medical necessity when it is otherwise normal, we encourage moral hazard. Second, there must be limits to moral obligation in medicine. Daniels illustrates this with his plain hero example. The plain hero thinks that he might perform better if he were beautiful, but society is entitled to disagree with his expensive tastes. For Daniels and others who hold that medical necessity is the barometer of moral obligation in medicine, enhancement is not necessarily futile. Rather, it goes beyond what institutions of medicine can reasonably be expected to accomplish given their emphasis on avoiding moral hazard and treating only medical conditions.

The third scheme for understanding enhancement is best articulated by Norman Daniels and John Rawls (see the article by Daniels in this Special Section), and amplifies the second scheme. Medicine has the role of restoring people to the status of "normal competitors," not "equal" competitors. Medicine is not required to make everyone equally happy. Amartya Sen and others argue that medicine may aim at eliminating disability, but Daniels's more restricted view is that medicine and medical practitioners are obliged to patients only to the point of restoring them to ordinary function. To define human function, Daniels and others have turned to Christopher Boorse's description of "species-typical" functioning. ${ }^{3}$

Boorse's is a very strong view: humans like all animals behave in measurable ways, and there must be an average for these behaviors and for the organic activity that undergirds behavior. There are indeed computations in the literature of biomedicine for averages of virtually every organic component, and for many behaviors, measured in particular populations at particular times. A normal competitor may have all sorts of disadvantages. But the disadvantages for any competitor do not rise to the level of medical necessity unless they can be shown to deviate from normal (speciestypical) functioning, and even then the obligation of medicine is only to return the normal competitor to the range of normal functioning, not to make the normal competitor an equal of every other competitor. Our functions as organisms exist beneath, and prior to, our capacities in human competitive endeavors.

The focus in this third scheme is on allowing talents, skills, and other capabilities to be distributed unequally, while preventing an unjust denial of medical care for conditions clearly 
understood to be disease related. Treating disease only when it involves a deviation from normal functioning is a way to avoid a number of economic and social problems, including the stigmatization of all the traits people long to eliminate through enhancement technology.

This is the most complex of the schemes for understanding enhancement, and it is also the most debated. There are a number of problems with the scheme-most importantly, its reliance on species-typical functioning. It can be argued that there are no nonnormative measures for the species' function, as any measure must rely on both the values of the investigator and on the data available in the particular population to be measured. Nor is there any clear let alone objective definition of function, itself a hotly debated notion through twentieth century biology. It is also entirely different to say that treatment is medically necessary than to say that a particular treatment is appropriate. There being the necessity to treat the shy bipolar does not suggest that the shy bipolar should be eligible for some particular treatment. Identification of the atypical person, functioning abnormally, tells us nothing about treatment for that person and thus nothing about the expense or modality that should be involved. Thus it will be difficult to use speciestypical or normal functioning analysis to reject what Daniels terms expensive tastes. It may be very expensive to bring the abnormal competitor back into the range of normal functioning after he shatters his leg, and might involve very exotic care at high cost. Normal functioning merely obscures and complicates cost issues.

It is also a problem of normal functioning that there is no focus on the upstream development of therapies and diagnostics. Research and the training of physicians play an important role in the development of the supposedly objective categories of normal function. Daniels does not refine the concept of enhancement outside the context of resource limits and equality, so we are left to wonder whether it would be objectionable for all competitors to be made equally better. Why shouldn't the plain hero have enhancement if he or she can pay for it and if the effects on opportunity are negligible.

The fourth scheme for distinguishing enhancement and treatment is the pragmatic approach. Articulated in these pages by John Lachs and elsewhere by myself and David Magnus, ${ }^{4}$ this approach to enhancement maintains that distinctions between treatment and enhancement must rely on strictly contextual accounts given by professionals and patients in their institutions. Disease and therapy are both defined by the institutions of medicine and their peer institutions, and are under explicit pressure of economic shortage and competing incentives. Local accounts of health have importance for determining what it means to cure or improve, and although there is explanatory value in the measurement of ordinary health and in definitions of normalcy, that value is in the service of human ends, not determinative of them. Will medical institutions have the capacity to incorporate enhancement technologies without losing the ability to deal with the myriad forces in play in micro- and macromedicine?

Some kinds of enhancement would give competitors special advantages. For these, there are questions about whether the games in which competitors participate can tolerate enhancement of specific individuals, or whether certain enhancements should be considered cheating. If we suppose, as does James Nelson, that schmoctors, not doctors, are performing enhancements, and ignore the problem of whether physi- 
cians should participate in enhancement, issues remain for discussion. A good example is the use of test training courses for college entrance exams. Some enhancements are not, or not primarily, about gaining special advantage in a known competition. These raise the problem of enhancement per se: Is there a problem with the development and sanction of cosmetic surgery, with the over-the-counter use of antidepressants by happy people or those who want to be happy but are not clinically depressed? What of medication for attention deficit hyperactivity disorder for those of us who just want to better focus on our work? Proponents of the fourth scheme hold that many clinical technologies and techniques present no clinical problem but instead call for social debate about the meaning of human flourishing. The goal of a pragmatic scheme is to direct the development of enhancements to human flourishing so that all those with access to such techniques are presented with safe options and are educated and thoughtful about their use. The appeal is not to equality but rather to tolerance and the satisfaction of deeply held needs of humans. The problem then becomes one of developing and choosing the right institutions, people, and pills to solve the problem.

In these pages we find a lively and up-to-date debate about what enhancement is and could be. Unlike many debates in bioethics, this one is being joined by a thoughtful discussion in the pages of many Internet sites and broad journals of public opinion. It has also been joined by massive corporate and research institutions, and in many ways portends the future and potential for public discussion of bioethics and the meaning of human flourishing.

\section{Notes}

1. Perens E, ed. Enhancing Human Traits: Ethical and Social Emplications. Washington, D.C.: Georgetown University Press, 1998.

2. Daniels N. Just Health Care. New York: Cambridge University Press, 1985.

3. Boorse C. On the distinction between disease and illness. Philosophy and Public Affairs 1975; 5(1):49-68.

4. McGee G. Parenting in an era of genetics. Hastings Center Report 1997;27(2):16-22. 